2011-04

Separate representations of dynamics in rhythmic and discrete movements: evidence from motor learning

Howard, lan

http://hdl.handle.net/10026.1/4800

10.1152/jn.00780.2010

Journal of Neurophysiology

American Physiological Society

All content in PEARL is protected by copyright law. Author manuscripts are made available in accordance with publisher policies. Please cite only the published version using the details provided on the item record or document. In the absence of an open licence (e.g. Creative Commons), permissions for further reuse of content should be sought from the publisher or author. 


\section{Separate representations of dynamics in rhythmic and discrete movements: evidence from motor learning}

Ian S. Howard, James N. Ingram and Daniel M. Wolpert

J Neurophysiol 105:1722-1731, 2011. First published 27 January 2011; doi:10.1152/jn.00780.2010

You might find this additional info useful...

Supplemental material for this article can be found at:

http://jn.physiology.org/content/suppl/2011/04/07/jn.00780.2010.DC1.html

This article cites 42 articles, 13 of which can be accessed free at:

http://jn.physiology.org/content/105/4/1722.full.html\#ref-list-1

Updated information and services including high resolution figures, can be found at:

http://jn.physiology.org/content/105/4/1722.full.html

Additional material and information about Journal of Neurophysiology can be found at:

http://www.the-aps.org/publications/jn

This infomation is current as of April 15, 2011. 


\title{
Separate representations of dynamics in rhythmic and discrete movements: evidence from motor learning
}

\author{
Ian S. Howard, James N. Ingram, and Daniel M. Wolpert \\ Computational and Biological Learning Laboratory, Department of Engineering, University of Cambridge, Cambridge, \\ United Kingdom
}

Submitted 14 September 2010; accepted in final form 26 January 2011

Howard IS, Ingram JN, Wolpert DM. Separate representations of dynamics in rhythmic and discrete movements: evidence from motor learning. J Neurophysiol 105: 1722-1731, 2011. First published January 27, 2011; doi:10.1152/jn.00780.2010.- Rhythmic and discrete arm movements occur ubiquitously in everyday life, and there is a debate as to whether these two classes of movements arise from the same or different underlying neural mechanisms. Here we examine interference in a motor-learning paradigm to test whether rhythmic and discrete movements employ at least partially separate neural representations. Subjects were required to make circular movements of their right hand while they were exposed to a velocity-dependent force field that perturbed the circularity of the movement path. The direction of the force-field perturbation reversed at the end of each block of 20 revolutions. When subjects made only rhythmic or only discrete circular movements, interference was observed when switching between the two opposing force fields. However, when subjects alternated between blocks of rhythmic and discrete movements, such that each was uniquely associated with one of the perturbation directions, interference was significantly reduced. Only in this case did subjects learn to corepresent the two opposing perturbations, suggesting that different neural resources were employed for the two movement types. Our results provide further evidence that rhythmic and discrete movements employ at least partially separate control mechanisms in the motor system.

interference; rhythmic movements; human

DURING EVERYDAY LIFE, we move our arms using rhythmic or discrete movements, or a combination of both, depending on the task. For example, to brush our teeth or beat and egg we use rhythmic movements, whereas to catch a ball or grasp a cup we use discrete movements. The relationship between these two classes of movements has been the topic of many studies (Buchanan et al. 2006; Ikegami et al. 2010; Miall and Ivry 2004; Schaal et al. 2004; Smits-Engelsman et al. 2002; Sternad 2000; Sternad and Dean 2003; van Mourik and Beek 2004). One view is that discrete movements represent the fundamental class and that rhythmic movements are merely a concatenation of a series of discrete movements. The opposing view is that rhythmic movements represent the fundamental class and that discrete movements are simply truncated rhythmic movements. Both of these viewpoints would suggest that only a single control mechanism is involved. A third viewpoint is that rhythmic and discrete movements represent two distinct movement classes that are mediated by separate neural control circuitry. Further support for this latter hypothesis has recently

Address for reprint requests and other correspondence: I. Howard, Computational and Biological Learning Laboratory, Dept. of Engineering, Univ. of Cambridge, Trumpington St., Cambridge CB2 1PZ UK (e-mail: ish22@cam. ac.uk). been obtained from behavioral (Ikegami et al. 2010) and imaging studies (Schaal et al. 2004).

Behavioral studies of motor adaptation to perturbations that examine interference and transfer provide a means to investigate the underlying partitioning of neural representations and control mechanisms. These studies typically use two different perturbations, which show interference in the absence of contextual cues (Bock et al. 2001; Brashers-Krug et al. 1996; Goedert and Willingham 2002; Karniel and Mussa-Ivaldi 2002; Krakauer et al. 2005; Krakauer et al. 1999; Miall et al. 2004; Shadmehr and Brashers-Krug 1997; Wigmore et al. 2002). When the perturbations are each associated with a different context, a reduction in this interference is often observed. In the case of discrete movements, several studies have shown a reduction in interference when each perturbation is associated with a unique context (Cothros et al. 2009; Howard et al. 2008; 2010; Krouchev and Kalaska 2003; Nozaki et al. 2006).

Several recent models have been developed to account for the reduction in interference observed in the presence of contextual cues (Lee and Schweighofer 2009; Nozaki and Scott 2009). These models propose multiple internal representations, which can be selectively engaged by different contexts (Wolpert and Kawato 1998). In the present study, we ask whether rhythmic and discrete-movement classes are associated with separate representations. At a neurophysiological level, context-dependent learning of opposing perturbations may involve different populations of neurons that are engaged by the different sensorimotor contexts (Nozaki et al. 2006). Similarly, when the perturbation remains constant, a lack of transfer from one context to another may imply some separation of the underlying representations.

With regard to rhythmic and discrete movements, Ikegami investigated transfer during motor learning of a visuomotor perturbation during point-to-point movements (Ikegami et al. 2010). They found incomplete transfer from rhythmic to discrete movements, suggesting that these two classes of movements may engage separate representations. Here we use a dynamic interference paradigm to investigate the representations associated with rhythmic circular and discrete circular movements. Specifically, subjects performed continuous circular movements consisting of multiple revolutions in the rhythmic case or discrete movements consisting of one and a quarter revolutions in the discrete case. We first verify that subjects adapt similarly to the dynamic perturbation for each movement class and that interference occurs when the perturbation direction is reversed. We then show that interference is reduced in a switching paradigm when each perturbation direction is 
associated with a different movement class. These results provide further evidence that rhythmic and discrete movements are distinct classes that engage at least partially separate neural representations.

\section{MATERIALS AND METHODS}

A total of 44 right-handed subjects took part in the study (2 were excluded from the final analysis as described below). Subjects provided written informed consent and were naïve to the aims of the experiment. The protocol was approved by the Cambridge Psychology Research Ethics Committee, and all subjects completed an Edinburgh handedness questionnaire.

All experiments were performed using a vBOT planar robotic manipulandum, with associated virtual reality C-rig and air table (Howard et al. 2009). The vBOT is a custom-built back-drivable planar robotic manipulandum that exhibits low mass at its handle. Position is measured using optical encoders sampled at $1,000 \mathrm{~Hz}$ and torque motors allow translational forces to be applied at the same rate. A virtual reality system was used to overlay target and cursor images in the plane of movement. Subjects were seated in a sturdy chair in front of the apparatus. They were firmly strapped against the backrest of the chair using a four-point seatbelt to reduce body movement. Each subject held the robot handle in his or her right hand, and the right forearm was supported by an air sled that constrained movements to the horizontal plane. Subjects were prevented from viewing their hands directly.

All experiments required subjects to perform either rhythmic or discrete clockwise circular movements around the circumference of a visually presented target circle (8-cm radius). The center of the target circle was located in the subject's midsagittal plane $\sim 30 \mathrm{~cm}$ below the eyes and $30 \mathrm{~cm}$ in front of the chest. The handle controlled a red cursor (disc of $0.5-\mathrm{cm}$ radius). At the start of each trial, the target circle and the start position for the movement appeared. The start position was a $1.0-\mathrm{cm}$ radius disc located on the circumference of the target circle. At the start of each trial the vBOT applied a force to the subject's hand, moving the cursor to the start position. A trial began when the cursor had remained within its start position at a speed below $0.1 \mathrm{~cm} / \mathrm{s}$ for $0.5 \mathrm{~s}$. Subjects were then cued to start the movement by an acoustic tone, which was used as a go signal. They were required to achieve the circular movement at a speed of 1 revolution/s. To assist this, a pair of visual indicators (pacing cursors) moved along the circumference of the target circle at the required frequency. These were short line segments perpendicular to the tangent, similar to the distal parts of the hands of a clock and separated by $180^{\circ}$. Subjects were informed that the moving cursors were only meant to act as a pacemaker for the speed of their movements and that they were not required to track them. To this end, a pair of pacing cursors (rather than a single cursor) was used to make it easier for subjects to pace their movement around the circle. Notably, using a pair of cursors discouraged subjects from tracking a single cursor. The cursors were present in both the discrete and rhythmic conditions.

Rhythmic and discrete movements. Subjects performed either continuous circular movements consisting of 20 revolutions in the rhythmic case or discrete movements consisting of $11 / 4$ revolutions in the discrete case. They performed blocks containing either a single rhythmic movement $(1 \times 20$ revolutions $)$ or 16 individual discrete movements $(16 \times 11 / 4=20$ revolutions $)$. For rhythmic movements the start position was always located at 12 o'clock on the circumference of the target circle. Subjects were required to make 20 revolutions continuously, without stopping in between. The experimental algorithm counted the number of revolutions and terminated the trial after 20 had been performed. For discrete movements, subjects were required to make single discrete circular movements of $11 / 4$ revolutions per trial, with a short pause $(\sim 6 \mathrm{~s})$ between each trial. The start and end positions for the movement were displayed on the circumference of the target circle. Specifically, at the beginning of each trial, the start position was displayed. After the movement had been initiated, the start position disappeared and was replaced by the end position (1 $1 / 4$ revolutions clockwise relative to the start position). The end position then became the start position for the next trial. At the beginning of each block, the start position was located at 12 o'clock (as for the rhythmic case), stepping 1/4 revolution increments around the target circle with each consecutive trial. Consequently, after 16 discrete movements, the end position coincided with the start position for the block. For both rhythmic and discrete cases, the subject's performance was monitored, and error messages were displayed if too many or too few rotations were performed. Subjects were also notified if their movements were too slow.

Perturbation. Each block of movements was either made in a null field (no forces) or with the vBOT generating one of two possible dynamic perturbations, which consisted of a viscous skew field (Shadmehr and Mussa-Ivaldi 1994):

$$
\left[\begin{array}{c}
F_{x} \\
F_{y}
\end{array}\right]=k\left[\begin{array}{ll}
0 & 1 \\
1 & 0
\end{array}\right]\left[\begin{array}{l}
\dot{x} \\
\dot{y}
\end{array}\right]
$$

where $k$ was set equal to $-8.5 \mathrm{~N} \mathrm{~m}^{-1} \mathrm{~s}$ (perturbation $\mathrm{P} 1$ ) or $+8.5 \mathrm{~N}$ $\mathrm{m}^{-1} \mathrm{~s}$ (perturbation $\mathrm{P} 2$ ). The perturbations $\mathrm{P} 1$ and $\mathrm{P} 2$ are illustrated in Fig. $1, A$ and $B$, respectively.

Catch trials and catch revolutions. In general, there are two ways of adapting to a state-dependent force field. First, subjects can simply cocontract the muscles, thereby stiffening the arm so that the positional displacement attributable to the experienced forces are reduced. Such a mechanism works for any force field and does not require the motor system to represent the particular structure of the field. Second, the subject can learn a mapping between the state of the limb and the force required to compensate for the force field. Such learning is specific for the particular field. Indeed, previous studies have suggested that, during exposure to a dynamic force field, it is not a virtual trajectory that is learned but rather a mapping from state to expected force, that is, a controller (Conditt et al. 1997). Cocontraction and specific compensation are not mutually exclusive, and a number of studies have examined their interaction (Tee et al. 2010). Our use of catch trials to distinguish between adaptation attributable to nonspecific cocontraction and adaptation attributable to specific compensation to the state-dependent force field is based on the standard method (Shadmehr and Mussa-Ivaldi 1994). To examine these components in our experiments, catch trials were used to examine the after-effects observed when the field was unexpectedly turned off. In this case, the two strategies lead to different predictions with respect to the presence of after-effects. If subjects use stiffness, then no after-effects should be seen. Specifically, in the absence of the force field, subjects simply make the normal movement with a stiffer arm. However, if specific compensation is used, subjects will initially produce a force that is inappropriate in the absence of the force field, causing the hand to deviate from the intended movement.

In the case of discrete movements, we included randomly presented catch trials in which the force field was turned off for an entire trial. In the case of rhythmic movements, one of the multiple revolutions was used to assess learning, and we refer to this as a catch revolution. During such a rhythmic-movement catch revolution, the force was rapidly ramped down (over $100 \mathrm{~ms}$ ) to zero for one revolution and then ramped up again. Ramping the forces in this manner avoided force discontinuities.

Error measures. Because subjects were asked to make circular movements, performance on each trial was defined with respect to the circularity of the hand path. The skew fields used for perturbations P1 and $\mathrm{P} 2$ have the effect of causing circular movements to deform into elliptical paths (see example in Fig. 2B). To analyze the paths, we used an algorithm that can reliably operate on scattered data to fit an ellipse (Fitzgibbon et al. 1999). We then define an error measure that is given by the ratio of the long axis to the short axis of the ellipse, from which unity is subtracted. The direction of the tilt induced by the 
Fig. 1. Viscous skew fields and circular movements. $A$ : viscous skew fields for perturbation P1. $B$ : viscous skew fields for perturbation P2. $C$ : elliptical deviation of a circular movement made in the viscous skew field P1 (in $A$ ). D: elliptical deviation of a circular movement made in the viscous skew field P2 (in $B$ ).
A
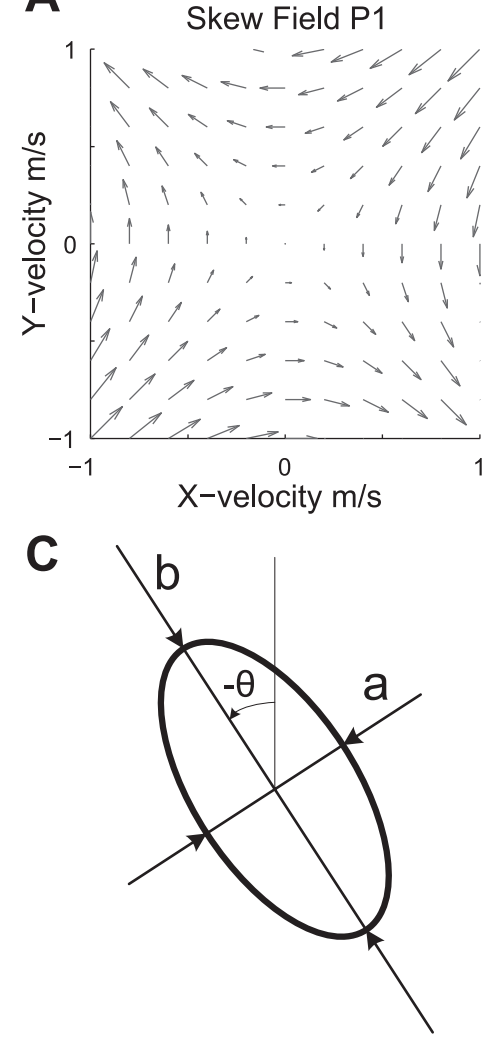

B

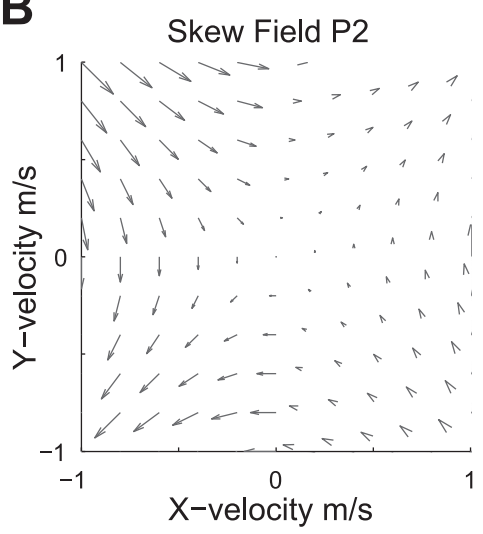

D

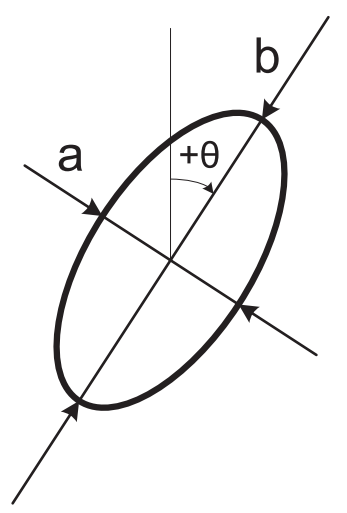

perturbation is then captured by the sign of the angle of the long axis relative to vertical, with a clockwise rotation taken as positive (Fig. 1, $C$ and $D)$. We refer to this measure as the signed error.

$$
\text { SignedError }=\operatorname{Sign}(\theta)(b / a-1)
$$

To quantify performance, we calculated the signed error over each entire block excluding data from catch trials (or revolutions) and points when the hand speed was less than $10 \mathrm{~cm} / \mathrm{s}$. For the discrete trails, the last $25 \%$ of data points of each trial was also excluded (to exclude terminal corrective movement to reach the final target position). Catch trials and catch revolutions were analyzed separately in the same way.

Statistical analysis. We perform only hypothesis-based planned comparisons and use repeated-measures ANOVA, which corresponds

\section{A}

\begin{tabular}{|c|c|c|c|c|c|c|c|c|c|c|c|c|c|c|}
\hline Perturbation & \multicolumn{2}{|c|}{ Null } & \multirow{2}{*}{$\frac{\text { P1 }}{\text { Rhythmic }}$} & \multirow{2}{*}{$\frac{\mathrm{P} 1}{\text { Rhythmic }}$} & \multirow{2}{*}{$\frac{\mathrm{P} 1}{\text { Rhythmic }}$} & \multirow{2}{*}{$\frac{\mathrm{P} 1}{\text { Rhythmic }}$} & \multirow{2}{*}{$\begin{array}{l}\cdot \cdot \bullet \\
\cdot \cdot \bullet\end{array}$} & \multirow{2}{*}{$\frac{\text { P2 }}{\text { Rhythmic }}$} & \multirow{2}{*}{$\frac{{ }^{\text {P2 }}}{\text { Rhythmic }}$} & \multirow{2}{*}{$\frac{\text { P2 }}{\text { Rhythmic }}$} & \multirow{2}{*}{$\frac{\text { P2 }}{\text { Rhythmic }}$} & \multirow{2}{*}{$\begin{array}{l}\bullet \cdot \bullet \\
\cdot \bullet \cdot\end{array}$} & \multicolumn{2}{|c|}{ Null } \\
\hline Movement & Rhythmic & Rhythmic & & & & & & & & & & & Rhythmic & Rhythmic \\
\hline Blocks & & & & & 28 & & & & & 28 & & & & \\
\hline
\end{tabular}

B

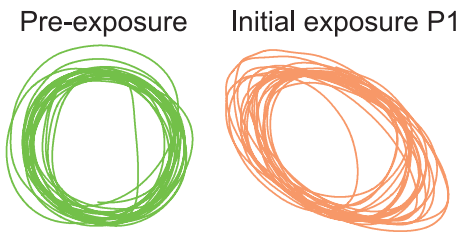

Initial exposure P2

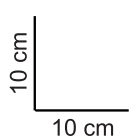

Final exposure P1

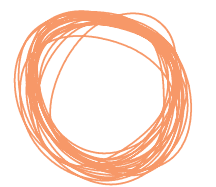

Final exposure P2

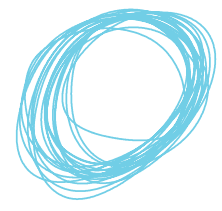

C

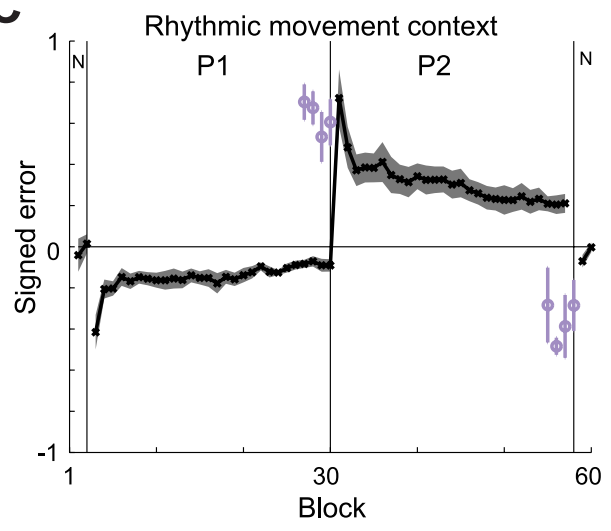

Fig. 2. Experiment 1: Static context: rhythmic movements. A: table showing the perturbation (P1 or P2), movement class (always rhythmic), and number of blocks for each perturbation. $B$ : paths for a single representative subject. Plot colors follow those used for the perturbations in $A$, and catch errors are shown in lavender (C). The upper row shows the paths during preexposure (null-field, block 2), initial exposure in P1 (block 3), and final exposure in P1 (block 30). The lower row shows the paths during initial exposure in P2 (block 31), final exposure in P2 (block 58), and postexposure (null-field, block 59). $C$ : signed mean error (solid line) and standard error (shading). Each point is the mean across subjects $(n=6)$. 
to a two-tailed $t$-test. We report uncorrected $P$ values across subjects to determine statistical significance of signed error. Although we perform a number of tests overall, only a few are truly critical for the conclusions of the paper, and these are in experiment 5, where we examine the postexposure after-effects.

Experiment 1: static-movement class: rhythmic. The purpose of experiment 1 was to confirm that subjects could learn the velocitydependent force fields while making rhythmic circular movements. The experiment is illustrated in Fig. 2A. A total of seven subjects participated in this experiment. However, one subject was excluded because of failure to produce consistent angular speed profiles across the trials, leaving six subjects in the final analysis $(n=6)$. The experiment included 60 blocks of rhythmic movements. The experiment consisted of an initial preexposure phase of two blocks in the null field (no forces), two consecutive exposure phases of 28 blocks each, and a final postexposure phase of two blocks again in the null field.

Half the subjects $(n=3)$ experienced viscous skew field P1 for the first exposure phase and viscous skew field $\mathrm{P} 2$ for the second exposure phase. For the other half of the subjects $(n=3)$, the order was reversed. This procedure was adopted to counterbalance any directional effects that may arise because of interaction with the biomechanics of the arm and to minimize any other effects of direction. During the last four blocks of each exposure phase, a single catch revolution was included pseudorandomly between the 12th and 18th revolution in each block.

Experiment 2: static-movement class: discrete. The purpose of experiment 2 was to confirm that subjects could learn the velocitydependent force fields while making discrete circular movements. A total of six subjects $(n=6)$ participated. The experiment was identical to experiment 1, except that subjects made discrete circular movements (Fig. 3A). In this case, each block consisted of 16 discrete trials, with each trial consisting of a circular movement of $11 / 4$ revolutions. During the last four blocks of each exposure phase, catch trials (null-field trials) were included and were pseudorandomly inserted between the 10th and 14th trials in each block.

Experiments 3-5: switching perturbation. In experiments 3-5 we used an interference paradigm to determine whether rhythmic and discrete movements involve separate control mechanisms. A total of
18 subjects participated. Subjects were exposed to opposing perturbations, which alternated across consecutive blocks so that oddnumbered blocks were associated with one perturbation direction and even-numbered blocks with the other (Fig. 4). In the first two experiments, subjects made either rhythmic movements (experiment 3, Fig. $4 A$ ) or discrete movements (experiment 4, Fig. $4 B$ ) while the perturbations alternated. In these experiments the class of movement remained static throughout. In experiment 5, subjects made rhythmic and discrete movements alternately across consecutive blocks so that each perturbation direction was associated with a different movement class (Fig. 4C).

Experiment 3: switching perturbation, static-movement class: rhythmic. In experiment 3 , six subjects $(n=6)$ performed 90 blocks of rhythmic movements (Fig. 4A). The experiment began with a preexposure phase consisting of two blocks in the null field, followed by an exposure phase of 86 blocks in which subjects experienced the alternating dynamic, viscous skew-field perturbations (P1 and P2, see Fig. 1, $A$ and $B$ ). Finally, subjects performed a postexposure phase consisting of two blocks in the null condition. As in experiment 1 , catch revolutions were included to assess learning during the last four exposure blocks.

Experiment 4: switching perturbation, static-movement class: discrete. Experiment 4 was identical to experiment 3, except that six subjects $(n=6)$ made 16 discrete movements per block, and catch trials were used to assess learning (Fig. 4B).

Experiment 5: switching perturbation and movement class: rhythmic/discrete. Experiment 5 was identical to experiment 3, except that subjects made rhythmic and discrete movements alternately across consecutive blocks (Fig. 4C). A total of seven subjects participated in this experiment. However, one subject was excluded because cursor movements made during the initial exposure block lay outside the normal workspace. For the six remaining subjects $(n=6)$, the perturbation direction was counterbalanced, so that half the subjects $(n=3)$ experienced perturbation P1 for odd-numbered blocks and perturbation P2 for even-numbered blocks. For the other half of the subjects $(n=3)$, the order was reversed.

Experiments 3-5: analysis. We performed an analysis that allowed us to compare both the static-movement experiments, which consisted of 90 blocks for each of the two movement classes, with the switch-

\begin{tabular}{|c|c|c|c|c|c|c|c|c|c|c|c|c|c|c|}
\hline Perturbation & \multicolumn{2}{|c|}{ Null } & P1 & P1 & P1 & P1 & - • • & P2 & P2 & P2 & P2 & - • • & \multicolumn{2}{|c|}{ Null } \\
\hline Movement & Discrete & Discrete & Discrete & Discrete & Discrete & Discrete & - • • & Discrete & Discrete & Discrete & Discrete & •・ & Discrete & Discrete \\
\hline Blocks & \multicolumn{2}{|c|}{2} & \multicolumn{5}{|c|}{28} & \multicolumn{5}{|c|}{28} & \multicolumn{2}{|c|}{2} \\
\hline
\end{tabular}

B

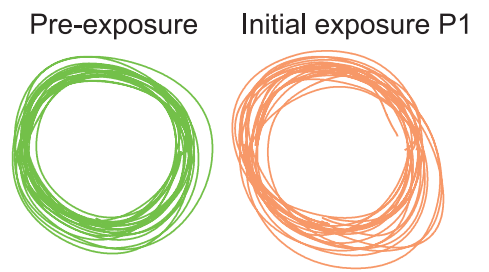

Initial exposure P2

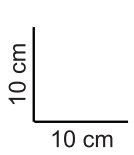

Final exposure P1

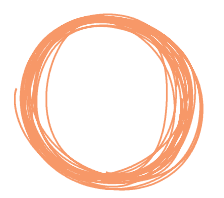

Final exposure $\mathrm{P} 2$

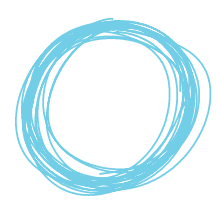

C

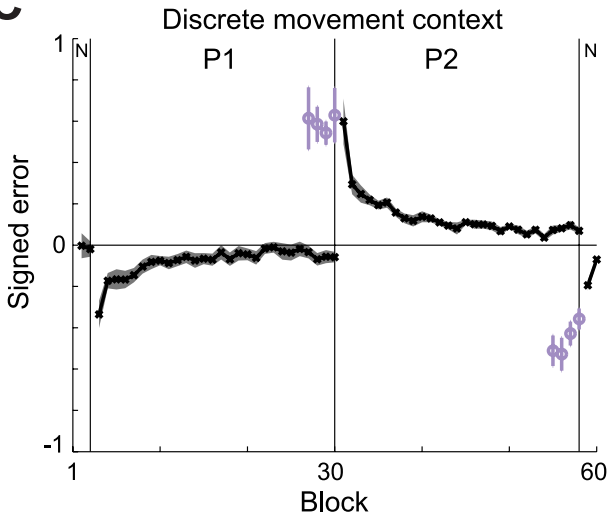

Fig. 3. Experiment 2: Static context: discrete movements. A: table showing the perturbation (P1 or P2), movement class (always discrete), and number of blocks for each perturbation. $B$ : paths for a single representative subject. Plot colors follow those used for the perturbations in $A$, and catch errors are shown in lavender $(C)$. The upper row shows the paths during preexposure (null-field, block 2), initial exposure in P1 (block 3) and final exposure in P1 (block 30). The lower row shows the paths during initial exposure in P2 (block 31), final exposure in P2 (block 58), and postexposure (null-field, block 59). $C$ : signed mean error (solid line) and standard error (shading). Each point is the mean across subjects $(n=6)$. 

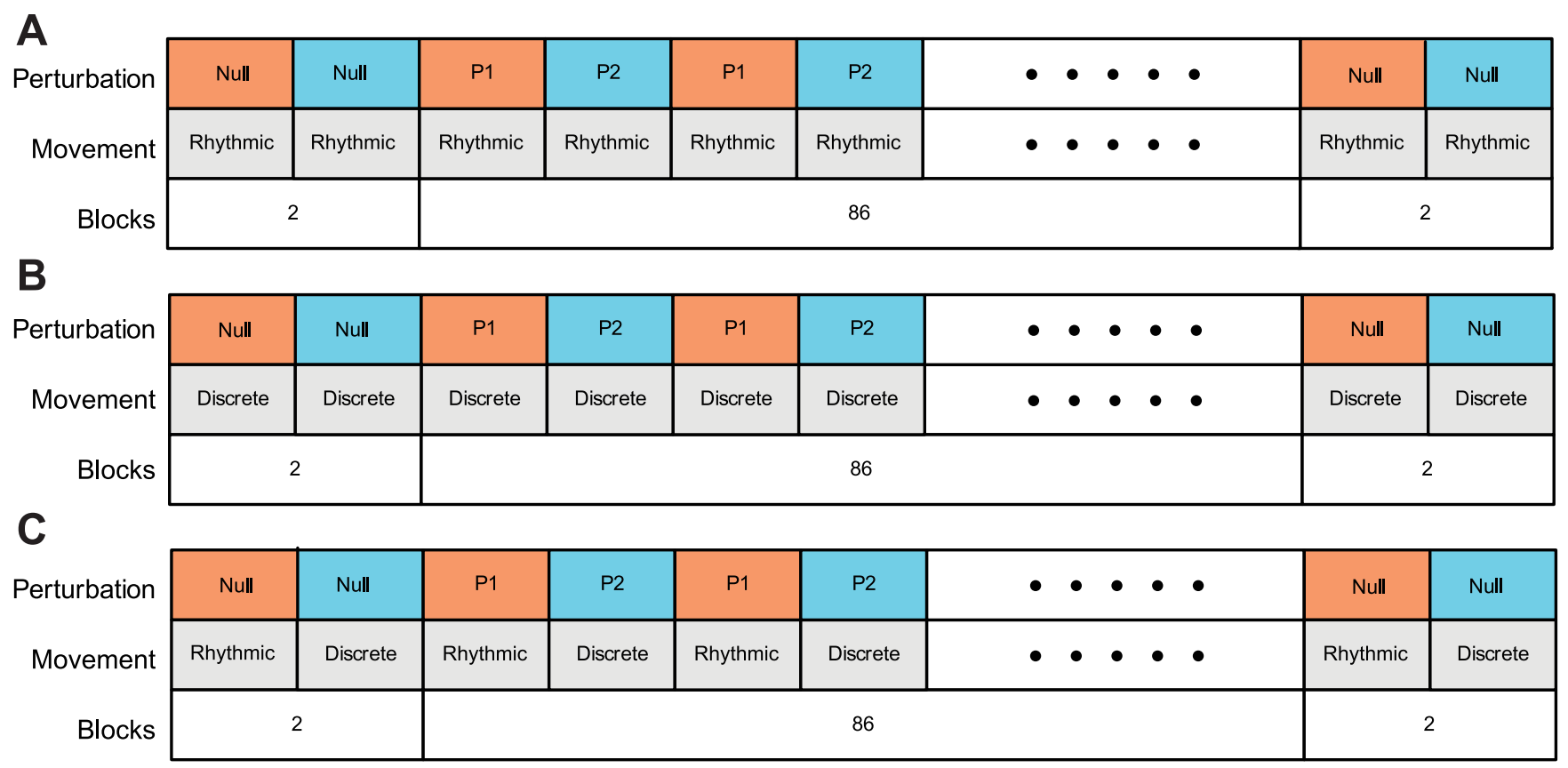

Fig. 4. Experiments 3-5: switching perturbations: paradigm. A: table showing perturbation (P1 or P2), movement class (always rhythmic), and block numbers in the switching perturbation static rhythmic movement experiment 3. B: table showing perturbation (P1 or P2), movement class (always discrete), and block numbers in the switching perturbation static-discrete movement experiment 4. $C$ : table showing perturbation (P1 or $\mathrm{P} 2)$, movement class (rhythmic or discrete), and block numbers in the switching perturbation, switching movement class experiment 5.

ing-movement experiment, which consisted of 45 blocks for each of the two movement classes. Specifically, for the two static-movement experiments, we computed the mean signed error over pairs of blocks, reducing the error time series from 90 blocks to 45 block pairs, one for each static-movement class (rhythmic and discrete). In this case, the sign of the error was adjusted to take into account the opposite perturbation directions associated with consecutive blocks. For the switching experiment, the signed error was computed separately for the blocks associated with each movement class. This yielded two time series consisting of 45 blocks, one for each switching-movement class. When calculating the average across subjects, we also adjusted the sign of the error according to the perturbation direction (which was counterbalanced across subjects as described above).

Control experiments. In the previous experiments, subjects were allowed to make movements without having to track the cursors, which were present simply to pace movement speed. A comparison of the discrete- and the rhythmic-movement speeds showed that they had slightly different ranges. To ensure that any effects from experiment 5 did not arise as a result of these small differences in speed, two control experiments were performed.

Experiment S1: switching perturbation and movement class: speed control. The control experiment $S 1$ (see supplemental material, which is available online on the Journal of Neurophysiology website) was similar to experiment 5, except that a modified pacing cursor was employed to ensure a closer match in speed.

Experiment 6: switching perturbation, static-movement class: twospeed control. Experiment 6 was a control experiment similar to experiment 3 , except that subjects $(n=6)$ were required to make rhythmic movements that alternated across successive blocks between rotational frequencies of $0.9 \mathrm{~Hz}$ and $1.1 \mathrm{~Hz}$. The speed differences were chosen to be roughly equivalent to the difference that we observed between the rhythmic and discrete cases in experiment 5 , to investigate whether such speed differences were sufficient to account for the observed learning (the speed profiles for these rhythmic and discrete cases from experiment 5 are shown in Fig. S2 in the supplemental material). As such, each perturbation direction was associated with rhythmic movements of a different frequency. This is in contrast to experiment 3 in which subjects made rhythmic movements at $1 \mathrm{~Hz}$ for both perturbations. To compensate for the reduction in speed between the two conditions, the field strength was increased at the lower rotational frequency and decreased at the higher rotational frequency. Specifically, viscous skew fields P1 and P2 employed gains of $k=-7.64 \mathrm{~N} \mathrm{~m}-1 \mathrm{~s}$ and $k=+9.33 \mathrm{~N} \mathrm{~m}-1 \mathrm{~s}$, respectively ( $E q$. 1). Histograms of hand velocity and position, as well as the mean and standard error of the speed profiles across subjects, were computed separately for the two rhythmic frequencies.

\section{RESULTS}

Static-movement-class experiments. In the first two experiments, subjects carried out a single movement class (either rhythmic or discrete) throughout the duration of each experiment. They experienced a viscous skew field in one direction for the first half of the experiment and in the opposite direction for the second half. To quantify performance, we used a measure of circularity in which larger magnitude values corresponded to more elliptical movements and the sign reflected the direction of the tilt of the long axis (see MATERIALS AND METHODS and Fig. 1, $C$ and $D$ ).

Experiment 1: static-movement class: rhythmic. Figure $2 B$ shows the paths for a representative subject at key stages of the experiment, and Fig. $2 C$ shows the signed error as a function of block, averaged across all subjects. During the preexposure phase, subjects made roughly circular movements characterized by a low error. Upon introduction of the first perturbation, the movement paths became elliptical as a result of the compressive and expansive nature of the field, and the error increased correspondingly. Over the course of the 28 exposure blocks, the movements became more circular and performance improved significantly (change in error from first to last exposure block $+0.32 \pm 0.08, P=0.009$ ). The next 28 blocks were performed with the opposite perturbation direction, which resulted in a change in the sign of the error (Fig. 2C). Imme- 
diately after the change in the perturbation, the magnitude of the error increased. As before, performance improved over the course of exposure $(-0.47 \pm-0.1, P=0.01)$. The magnitude of the error on the first block in the new perturbation was significantly larger than the corresponding block in the first perturbation $(0.31 \pm 0.09$ higher, $P=0.02)$. This suggests that there is anterograde interference between the two perturbations for rhythmic circular movements, consistent with previous studies showing interference during point-to-point movements (Shadmehr and Brashers-Krug 1997).

To examine whether the reduction in error could have arisen solely from cocontraction, we used catch revolutions in which the perturbation was transiently turned off for a single revolution chosen randomly during the last four blocks of each perturbation. During the catch revolutions (Fig. $2 C$, lavender circles), the errors were in the opposite direction to the perturbation and were significantly different from zero for the first (mean $0.63 \pm 0.07, P<0.001$ ) and second (mean $-0.36 \pm$ $0.07, P=0.004)$ perturbations. These results suggest that subjects were specifically compensating for each particular perturbation.

Experiment 2: static-movement class: discrete. The second experiment was identical to the first except that subjects made discrete movements throughout. Figure $3 B$ shows the paths for a representative subject, and Fig. $3 C$ shows the signed error as a function of block, averaged across all subjects. The features in these plots are similar to the first experiment. Upon introduction of the first perturbation, the movement paths became elliptical because of the compressive and expansive nature of the field, and the error increased correspondingly. Over the course of the 28 exposure blocks, the movements became more circular and performance improved significantly (change in error from first to last exposure block $+0.28 \pm 0.06, P=$ 0.004). The next 28 blocks were performed with the opposite perturbation direction, which resulted in a change in the sign of the error (Fig. $3 \mathrm{C}$ ). Immediately after the change in the perturbation, the magnitude of the error increased. However, as before, performance improved over the course of exposure $(-0.53 \pm 0.11, P=0.006)$. The magnitude of the error on the first block in the new perturbation was significantly larger than the corresponding block in the first perturbation $(0.26 \pm 0.09$ higher, $P=0.04)$. This suggests that there is anterograde interference between the two perturbations for discrete circular movements. As with the first experiment, random catch trials were presented during the last four blocks of each perturbation. Catch-trial errors (Fig. 3C, lavender circles) were significantly different from zero for both the first (mean $=0.59 \pm 0.09, P=$ 0.002 ) and second (mean $=-0.46 \pm 0.06, P<0.001$ ) perturbations, suggesting that subjects were specifically compensating rather than cocontracting.

Experiments 3-5: switching perturbation. Experiments 3-5 constitute the main results from the present study. They were run to determine whether interference between opposing perturbations would be reduced by associating each perturbation with a different movement class. This would suggest that the control mechanisms associated with each movement class were at least partially separate. In these experiments, subjects were exposed to switching perturbations (P1 and P2) in which the perturbation direction alternated across successive blocks (Fig. 4).

Figure 5, $A-C$, shows the movement paths for representative subjects at key stages for the three experiments (the two static-movement-class experiments and the switching-movement-class experiment). Following the color code from Fig. 4, the paths from odd blocks are plotted in red, and the paths from even blocks are plotted in blue. Figure $5 D$ shows the signed error for all three experiments. Note that the signed error for the four experimental conditions is plotted with an arbitrary
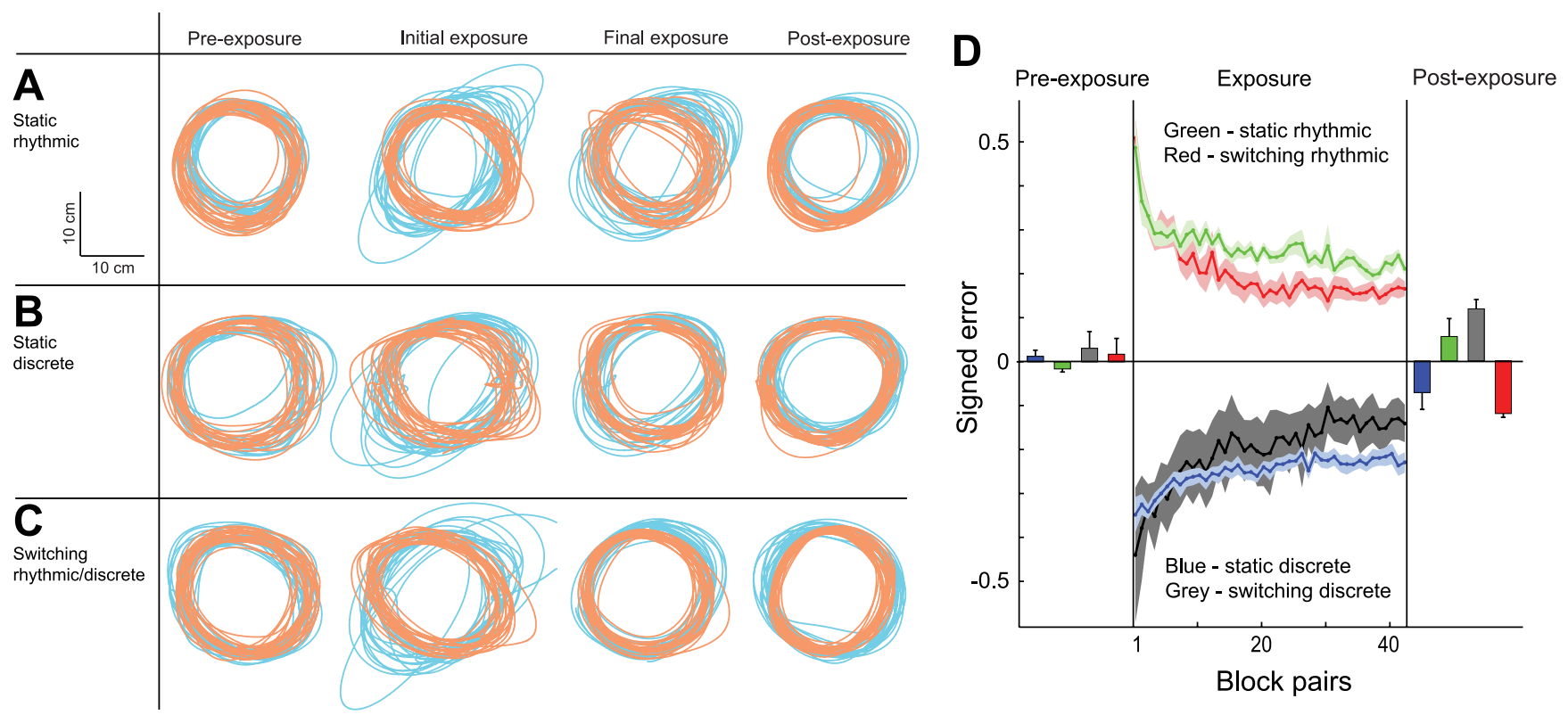

Fig. 5. Experiments 3-5: switching perturbations: results. A: paths for the static-rhythmic movement experiment 3 for a single representative subject showing odd (red) and even blocks (blue). The plot shows the paths during preexposure (blocks 1 and 2), initial exposure in P1 and P2 (blocks 3 and 4), final exposure in P1 and P2 (blocks 87 and 88), and postexposure (blocks 89 and 90). B: paths for the static-discrete movement experiment 4, plotted as in $A$. $C$ : paths for the switching rhythmic-discrete movement experiment 5, plotted as in A. D: signed mean error (solid line) and standard error (shading) for experiments $3-5$. Each point is the mean across subjects ( $n$ =6). The static-rhythmic movements from experiment 3 are plotted in green. The switching-rhythmic movements from experiment 5 are plotted in red. The static-discrete movements from experiment 4 are plotted in blue. The switching-discrete movements from experiment 5 are plotted in gray. 
sign to facilitate the presentation. Each condition generated errors in both directions (as within each experiment the field directions were counterbalanced), and these were combined in an appropriate fashion (see MATERIALS AND METHODS).

During the preexposure phase in the null field of each experiment, subjects made roughly circular movements characterized by a low signed error (preexposure bar chart at left of Fig. 5D; see figure legend for key to colors). Upon introduction of the perturbation, signed error increased. In Fig. 5, $A-C$, the errors are larger for one perturbation than the other because of the interaction of the biomechanical properties of the arm and the direction of the perturbation. The signed error reduced over the course of exposure in all cases but with important differences between the static and switching cases, which are described in detail below.

Experiment 3: switching perturbation, static-movement class: rhythmic. In the static rhythmic experiment (Fig. 5D, green curve) signed error decreased significantly between the first and last exposure block pairs $(-0.28 \pm 0.06, P=0.005)$. The mean signed error during catch revolutions was also significantly greater than zero $(0.24 \pm 0.03, P=0.0003)$. However, the signed error during the first postexposure block (green bar on right of Fig. $5 D$ ) was not significantly different from zero $(-0.06 \pm 0.04, P=0.23)$. The results from the postexposure block suggest that the improvement in performance was dominated by cocontraction. However, the catch revolutions indicate that some limited specific compensation was present within each block.

Experiment 4: switching perturbation, static-movement class: discrete. In the static-discrete experiment (Fig. 5D, blue curve) signed error also decreased significantly between the first and last exposure block pairs $(0.12 \pm 0.05, P=0.05)$. However, in this case the mean signed error during catch trials was not significantly different from zero $(-0.08 \pm 0.04, P=0.11)$. Similarly, the signed error during the first postexposure block (blue bar on right of Fig. 5D) did not differ significantly from zero $(0.07 \pm 0.04, P=0.12)$. The results from catch trials and the postexposure block suggest that the improvement in performance was attributable primarily to cocontraction with no evidence for the presence of specific compensation within each block.

Experiment 5: switching perturbation and movement class: rhythmic/discrete. In the rhythmic blocks of the switchingmovement-class experiment (Fig. $5 D$, red curve) signed error decreased significantly between the first and last exposure blocks $(0.34 \pm 0.0 .05, P<0.001)$. The mean signed error during catch revolutions was negative and significantly different from zero $(-0.358 \pm 0.1, P=0.02)$. In addition, the signed error during the postexposure block (red bar on right of Fig. $5 D$ ) was also negative and significantly different from zero $(0.12 \pm 0.01, P<0.001)$. A similar pattern was observed for the discrete movement blocks of this experiment (Fig. $5 D$, gray curve). Specifically, signed error decreased significantly between the first and last exposure blocks $(0.30 \pm 0.11, P=$ $0.047)$. The mean signed error during catch trials $(0.30 \pm 0.06$, $P=0.004$ ) and during the postexposure block (gray bar on right of Fig. $5 F, 0.12 \pm 0.02, P=0.003$ ) were both positive and significantly different from zero. Importantly, these aftereffects were in the opposite direction to the errors because of the perturbations. Thus, in contrast to the static-movementclass experiments (experiments 3 and 4, described above), the significant postexposure after-effects suggest that the improvement in performance in this case was attributable to specific compensation for the perturbations associated with each movement class. This is consistent with partially separate control mechanisms for rhythmic and discrete movements, which would allow the partitioning of perturbation-specific learning for each movement class.

Control experiments. It is possible that the improvement in performance observed for the switching-movement-class experiment (experiment 5, above) may have resulted from differences in the kinematics between the rhythmic and discrete movements rather than differences in the underlying control mechanisms. Two control experiments were therefore performed to examine whether such kinematic differences could explain the results.

Experiment $S 1$ (see supplemental material) was identical to experiment 5 , except that the speed at which subjects made the circular movements was more strictly controlled. Results show that this did not affect the degree of adaptation in the switching condition (Fig. S3).

Experiment 6: switching perturbation, static-movement class: two-speed control. Experiment 6 was an additional control experiment to examine the effects of differences in speed on the ability to learn the opposing perturbations. In this case, rather than associating each perturbation direction with a different movement class (as in the original experiment 5), each was associated with a different speed of rhythmic rotation $(1.1 \mathrm{~Hz}$ and $0.9 \mathrm{~Hz})$. These values were chosen to correspond to values observed during experiment 5 , and the differences were perceived as quite substantial by the subjects (see MATERIALS AND METHODS for further detail). Figure $6, A$ and $B$, show the velocity and position distributions, respectively, for this experiment. The different speeds of rotation can be clearly seen in the velocity distribution plots (Fig. 6A), whereas the position distributions are quite similar (Fig. 6B). This can also be appreciated in the mean speed profiles, which are shown in Fig. $6 C(\mathrm{red}=1.1 \mathrm{~Hz}$, blue $=0.9 \mathrm{~Hz})$. We compared the average speed during the plateau regions between the two speed conditions across subjects and found a significant difference $(8.2 \pm 1.1$ $\mathrm{cm} / \mathrm{s}, P<0.001)$. Note that this speed difference is comparable to that observed between the rhythmic- and discrete-movement classes of the switching experiment 5.

Figure $6 D$ compares the signed error for the static rhythmic experiment (red; experiment 3, above) with the two-speed control experiment (green). As can be seen, the plots are very similar for both experiments. Specifically, there were no significant differences for the final exposure errors between the two experiments $(-0.03 \pm 0.05, P=0.54)$. Moreover, as for the original static-rhythmic experiment, the signed error during the postexposure block did not differ significantly from zero $(-0.055 \pm 0.04, P=0.22)$. These results show that differences in rotational speed that were comparable to those observed in the switching-movement-class experiment were insufficient to allow subjects to corepresent the opposing perturbations.

\section{DISCUSSION}

We used a motor-learning-interference paradigm to examine the representations involved in the control of rhythmic and discrete circular arm movements. For comparison with previ- 

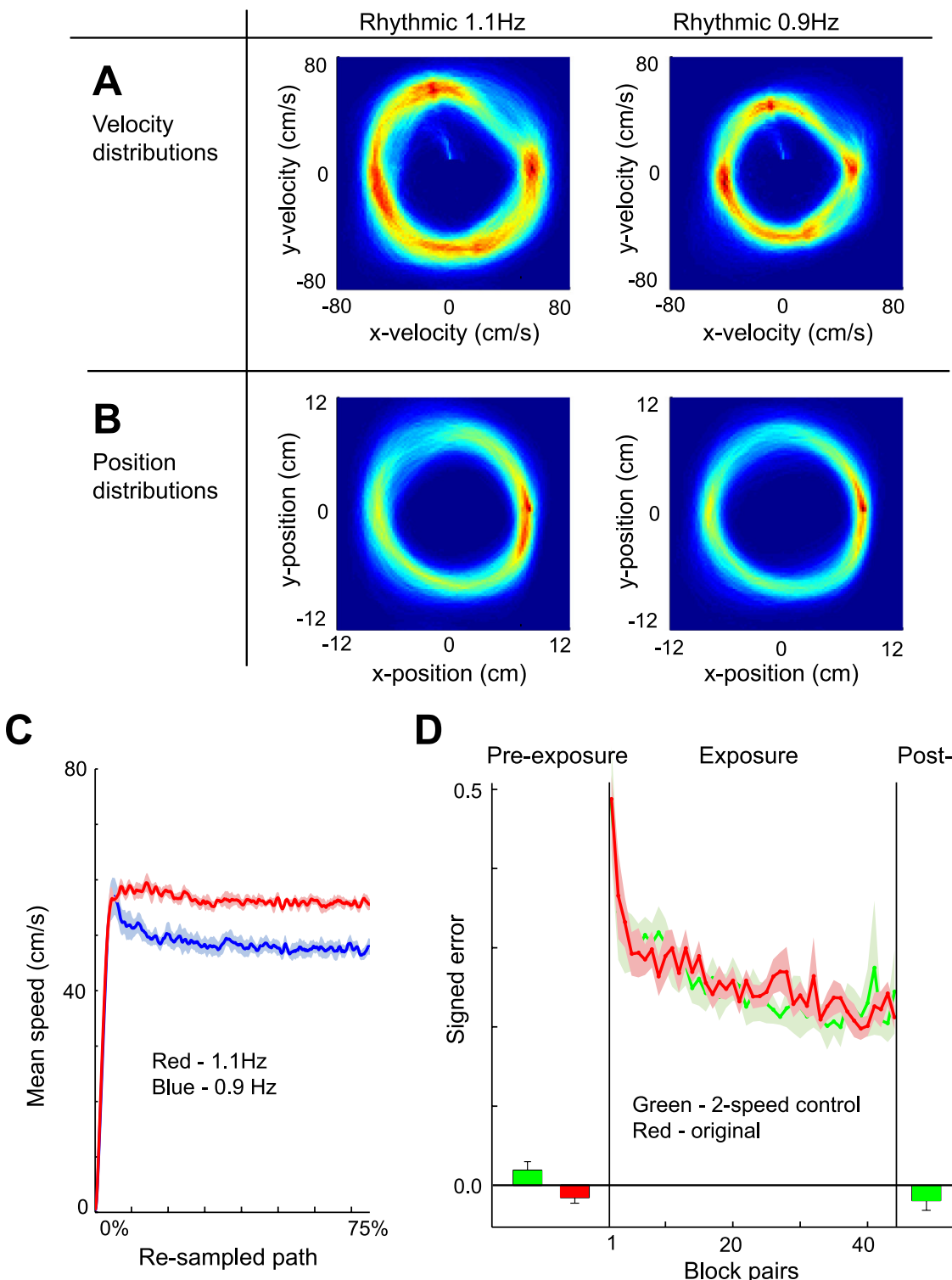

D

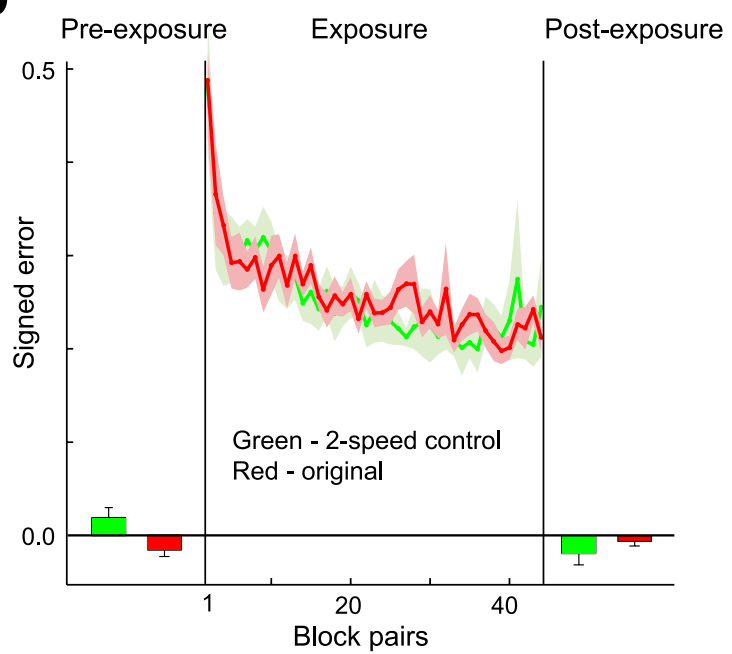

Fig. 6. Experiment 6: switching perturbation, static-movement class: two-speed control. $A$ : distributions of end-point velocity for the $1.1-\mathrm{Hz}$ (first column) and $0.9-\mathrm{Hz}$ (second column) rotation speed conditions across all trials and all subjects for the two-speed control experiment 6. $B$ : distributions of end-point position for the $1.1-\mathrm{Hz}$ (first column) and $0.9-\mathrm{Hz}$ (second column) rotation speed conditions across all trials and all subjects for the experiment 6. C: mean speed and standard error for the duration of the trial, normalized for trial length, across all trials and all subjects for the $1.1-\mathrm{Hz}$ (red) and $0.9-\mathrm{Hz}$ (blue) conditions. $D$ : signed mean error (solid line) and standard error (shading) for the two-speed control experiment 6 (green) and for the static-rhythmic movement experiment 3 (red). Each point is the mean across all subjects $(n=6)$. ous point-to-point reaching studies, we first confirmed the presence of anterograde interference when the direction of a dynamic force field reversed, during either rhythmic or discrete circular movements. We then examined a condition in which subjects were exposed to a dynamic force field whose direction reversed across successive blocks. In two experiments, subjects performed only rhythmic or only discrete movements while the perturbation direction reversed with each block. In both cases, performance improved over the course of the experiment, but null-field catch and postexposure trials suggested that this primarily arose through cocontraction rather than specific learning of the force fields. However, when each force-field direction was associated with a different movement class (either rhythmic or discrete) performance improved, with catch trials and postexposure after-effects demonstrating that subjects had learned to compensate specifically for the particular perturbation associated with each movement class. These results suggest that the control mechanisms for rhythmic and discrete movements are at least partially separate.
As pointed out by Hogan and Sternad (2007), in many previous studies the meanings of the terms rhythmic and discrete have not been defined precisely. To address this problem, they have proposed rigorous taxonomic definitions to describe different classes of movement. Their definitions are mathematically precise, including several subclassifications for both rhythmic and discrete movement types. They propose that the key feature of discrete movements is that they consist of a movement between starting and ending postures (of the endeffector) with a static holding phase before and after the movement. In contrast, the term rhythmic is used to cover a wide range of cyclic or periodic movements. In particular, they subdivide rhythmic movements on the basis of the regularity of their periodicity, which range from strictly and almost periodic, through quasiperiodic and transiently periodic, to recurrent and repetitive movements. They note that repetitive discrete movements can also fall within the definition of rhythmic movements, and these categories are not mutually exclusive. To further distinguish these classes, they also consider the smoothness of movement. Thus, whereas some repetitive discrete 
movements may be classed as rhythmic, they are often not as smooth as continuous rhythmic movements because they include discontinuities (nonzero high-order derivatives) in their movement paths introduced at their stopping and starting points.

In our study, we chose our two movement conditions to be distinct classes, as defined by Hogan and Sternad (2007). Our discrete movements consisted of circular rotations between start and end positions, with a static holding phase of $\sim 6 \mathrm{~s}$ between each movement. In addition, the start and end positions changed between each movement, thus minimizing cyclic repetitions. In contrast, we chose our rhythmic movements to be multiple continuous rotations with no pauses between revolutions. These movements were smooth, (almost) periodic (that is, as periodic as subjects were able to make), and consequently quite unlike repetitive discrete movements.

A recent study examined transfer of visuomotor learning between rhythmic and discrete movements (Ikegami et al. 2010). In one experiment, subjects were exposed to visuomotor perturbations while first performing either a set of discrete movements followed by a set of rhythmic movements or vice versa. The study reports almost complete transfer of learning from discrete to rhythmic movements but very little transfer from rhythmic to discrete movements, suggesting differences between the two movement classes. In a second experiment, multiple sets of rhythmic movements were performed in a visuomotor perturbation with delays between them. They found that, at the beginning of each rhythmic movement from the second set onward, errors were large for the first cycle, decreasing rapidly on subsequent cycles. In addition, when discrete training was employed with a large delay between trials, transfer to rhythmic movements was high for the first cycle but then degraded for the next few cycles before subsequently improving again. They hypothesized that this implies that the first cycle of the rhythmic movement engages control mechanisms associated with discrete movements. Overall, they draw the conclusion that different neural control mechanisms are employed for rhythmic and discrete movements. The present study is complementary to the Ikegami study in two ways. First, we investigated the learning of dynamic perturbations rather than kinematic transformations. Second, we used interference to probe the representations associated with the two movement classes. Thus the present study supports the hypothesis made by Ikegami that different neural control mechanisms are employed for rhythmic and discrete movements.

Previous studies have explored other contextual cues that allow opposing perturbations to be concurrently represented and have focused on discrete point-to-point movements (Cothros et al. 2009; Howard et al. 2008; 2010; Krouchev and Kalaska 2003; Nozaki et al. 2006). For example, in the case of dynamics, with extensive training, color can provide a contextual cue (Krouchev and Kalaska 2003). In addition, it has been shown that subjects are able to switch between a dynamic perturbation and a null field on the basis of visual feedback relating to grasp (Cothros et al. 2009). Another study showed that associating opposing perturbations with unimanual and bimanual movements allowed subjects to concurrently represent the perturbations (Nozaki et al. 2006). Likewise, opposing perturbations can be learned during bimanual movements under different contexts such as the hands acting on a single or two separate objects (Howard et al. 2008) or moving in the same or different directions (Howard et al. 2010). In all these bimanual studies, modulation of the motor representation associated with one limb appears to depend on the action (or absence of action) performed by the opposite limb. This phenomenon requires active movement because interference still occurs in the case where one arm is moved passively (Howard et al. 2010). Importantly, in this case all other potential cues except for active movement were the same. This suggests that, in the present study, the contextual cue must be the type of movement that is performed (rhythmic vs. discrete), rather than block identity.

Evidence that there is separate circuitry for rhythmic and discrete movements is consistent with recent neuroimaging studies. For example, Schaal performed functional MRI experiments while subjects made discrete or rhythmic movements, which consisted of single wrist-joint flexion/extension (Schaal et al. 2004). Their principle finding was that rhythmic movements activated a small number of unilateral primary motor areas, whereas discrete movement activated additional motor areas and showed strong bilateral activity in both cerebrum and cerebellum. They concluded that this provides evidence for separate control circuitry for discrete and rhythmic movements.

Several researchers have taken a more theoretical view when interpreting rhythmic and discrete movements. For example, rhythmic movements have been modeled using dynamical system theory, and such models can replicate many observed phenomena in both unimanual and bimanual movements, such as the stability of preferred phases and the transitions toward stable modes (Haken et al. 1985; Kay et al. 1987; Kelso 1984). A dynamical system analysis has also been extended to account for discrete and rhythmic movements. Schoner proposed that the dynamical systems perspective for understanding rhythmic movements could be generalized to account for discrete movements (Schoner 1990). He showed that a dynamical system model can exhibit discrete as well as continuous behaviors, using fixed-point and limit-cycle attractors, respectively. Recently, discrete movements have been modeled within the framework of optimal feedback control (Todorov and Jordan 2002). Although dynamical systems and optimal control have traditionally been considered separately, a framework has been proposed that unifies them, allowing a single model to account for both rhythmic and discrete movements (Schaal et al. 2007).

Several other computational models have also been proposed to account for both discrete and rhythmic movements (Ronsse et al. 2009; Ronsse et al. 2010). The implication is that these movement types constitute different movement primitives, and, in the case of combined movements, combinations of such primitives can occur. In most experimental studies, rhythmic and discrete arm movements are often studied in isolation, with little or no consideration to their interactions. Exceptions to this are found in studies involving the combination of discrete and rhythmic elements within movements (Staude et al. 2002; Sternad et al. 2002; Sternad et al. 2000). Such studies indicate that interaction occurs. For example, Sternad found that kinematic constraints for the coupling of discrete and rhythmic elements have a tendency to synchronize (Sternad et al. 2000). This was also the case for movements involving multi-joint coordination (Sternad and Dean 2003).

In conclusion, results from the present study show that associating opposing dynamic perturbations with rhythmic- 
and discrete-movement classes allows subjects to corepresent each perturbation and thereby learn dynamic perturbations, which would otherwise interfere. Importantly, adaptation to the particular perturbation associated with each movement class was accompanied by large postexposure after-effects, which were specific for the perturbation, indicating predictive compensation. In contrast, postexposure after-effects were not present in the static-context experiments, indicating that the reduction in error in this condition mainly arose because of cocontraction. Overall, these results are consistent with the existence of control mechanisms that are at least partially separate for rhythmic- and discrete-movement classes.

\section{GRANTS}

This study was supported by the Wellcome Trust and The European Project (SENSOPAC IST-2005-028056).

\section{DISCLOSURES}

The authors declare that they have no financial, personal, or professional interests that could be construed to have influenced the paper.

\section{REFERENCES}

Bock O, Schneider S, Bloomberg J. Conditions for interference versus facilitation during sequential sensorimotor adaptation. Exp Brain Res 138: 359-365, 2001.

Brashers-Krug T, Shadmehr R, Bizzi E. Consolidation in human motor memory. Nature 382: 252-255, 1996.

Buchanan JJ, Park JH, Shea CH. Target width scaling in a repetitive aiming task: switching between cyclical and discrete units of action. Exp Brain Res 175: 710-725, 2006.

Conditt MA, Gandolfo F, Mussa-Ivaldi FA. The motor system does not learn the dynamics of the arm by rote memorization of past experience. $J$ Neurophysiol 78: 554-560, 1997.

Cothros N, Wong J, Gribble PL. Visual cues signaling object grasp reduce interference in motor learning. J Neurophysiol 102: 2112-2120, 2009.

Fitzgibbon A, Pilu M, Fisher RB. Direct least square fitting of ellipses. IEEE Trans Pattern Anal Mach Intell 21: 476-480, 1999.

Goedert KM, Willingham DB. Patterns of interference in sequence learning and prism adaptation inconsistent with the consolidation hypothesis. Learn Mem 9: 279-292, 2002.

Haken H, Kelso JA, Bunz H. A theoretical model of phase transitions in human hand movements. Biol Cybern 51: 347-356, 1985.

Hogan N, Sternad D. On rhythmic and discrete movements: reflections, definitions and implications for motor control. Exp Brain Res 181: 13-30, 2007.

Howard IS, Ingram JN, Wolpert DM. Composition and decomposition in bimanual dynamic learning. J Neurosci 28: 10531-10540, 2008.

Howard IS, Ingram JN, Wolpert DM. Context-dependent partitioning of motor learning in bimanual movements. J Neurophysiol 104: 2082-2091, 2010.

Howard IS, Ingram JN, Wolpert DM. A modular planar robotic manipulandum with end-point torque control. J Neurosci Meth 181: 199-211, 2009.

Ikegami T, Hirashima M, Taga G, Nozaki D. Asymmetric transfer of visuomotor learning between discrete and rhythmic movements. J Neurosci 30: 4515-4521, 2010.

Karniel A, Mussa-Ivaldi FA. Does the motor control system use multiple models and context switching to cope with a variable environment? Exp Brain Res 143: 520-524, 2002.

Kay BA, Kelso JA, Saltzman EL, Schoner G. Space-time behavior of single and bimanual rhythmical movements: data and limit cycle model. $J$ Exp Psychol 13: 178-192, 1987.
Kelso JA. Phase transitions and critical behavior in human bimanual coordination. Am J Physiol Regul Integr Comp Physiol 246: R1000-R1004, 1984.

Krakauer JW, Ghez C, Ghilardi MF. Adaptation to visuomotor transformations: consolidation, interference, and forgetting. J Neurosci 25: 473-478, 2005.

Krakauer JW, Ghilardi MF, Ghez C. Independent learning of internal models for kinematic and dynamic control of reaching. Nat Neurosci 2: 1026-1031, 1999.

Krouchev NI, Kalaska JF. Context-dependent anticipation of different task dynamics: rapid recall of appropriate motor skills using visual cues. $J$ Neurophysiol 89: 1165-1175, 2003.

Lee JY, Schweighofer N. Dual adaptation supports a parallel architecture of motor memory. J Neurosci 29: 10396-10404, 2009.

Miall RC, Ivry R. Moving to a different beat. Nat Neurosci 7: 1025-1026, 2004.

Miall RC, Jenkinson N, Kulkarni K. Adaptation to rotated visual feedback: a re-examination of motor interference. Exp Brain Res 54: 201-210, 2004.

Nozaki D, Kurtzer I, Scott SH. Limited transfer of learning between unimanual and bimanual skills within the same limb. Nat Neurosci 9: 13641366, 2006.

Nozaki D, Scott SH. Multi-compartment model can explain partial transfer of learning within the same limb between unimanual and bimanual reaching. Exp Brain Res 194: 451-463, 2009.

Ronsse R, Sternad D, Lefevre P. A computational model for rhythmic and discrete movements in uni- and bimanual coordination. Neural Comput 21: 1335-1370, 2009.

Ronsse R, Wei K, Sternad D. Optimal control of a hybrid rhythmic-discrete task: the bouncing ball revisited. J Neurophysiol 103: 2482-2493, 2010.

Schaal S, Mohajerian P, Ijspeert A. Dynamics systems vs. optimal control-a unifying view. Prog Brain Res 165: 425-445, 2007.

Schaal S, Sternad D, Osu R, Kawato M. Rhythmic arm movement is not discrete. Nat Neurosci 7: 1137-1144, 2004.

Schoner G. A dynamic theory of coordination of discrete movement. Biol Cybern 63: 257-270, 1990.

Shadmehr R, Brashers-Krug T. Functional stages in the formation of human long-term motor memory. J Neurosci 17: 409-419, 1997.

Shadmehr R, Mussa-Ivaldi FA. Adaptive representation of dynamics during learning of a motor task. J Neurosci 14: 3208-3224, 1994.

Smits-Engelsman BC, Van Galen GP, Duysens J. The breakdown of Fitts' law in rapid, reciprocal aiming movements. Exp Brain Res 145: 222-230, 2002.

Staude G, Dengler R, Wolf W. The discontinuous nature of motor execution II. Merging discrete and rhythmic movements in a single-joint system-the phase entrainment effect. Biol Cybern 86: 427-443, 2002.

Sternad D. Debates in dynamics: a dynamical systems perspective on action and perception. Hum Mov Sci 19: 407-423, 2000.

Sternad D, de Rugy A, Pataky T, Dean WJ. Interaction of discrete and rhythmic movements over a wide range of periods. Exp Brain Res 147: 162-174, 2002.

Sternad D, Dean WJ. Rhythmic and discrete elements in multi-joint coordination. Brain Res 989: 152-171, 2003.

Sternad D, Dean WJ, Schaal S. Interaction of rhythmic and discrete pattern generators in single-joint movements. Hum Mov Sci 19: 627-664, 2000.

Tee KP, Franklin DW, Kawato M, Milner TE, Burdet E. Concurrent adaptation of force and impedance in the redundant muscle system. Biol Cybern 102: 31-44, 2010.

Todorov E, Jordan MI. Optimal feedback control as a theory of motor coordination. Nat Neurosci 5: 1226-1235, 2002.

van Mourik AM, Beek PJ. Discrete and cyclical movements: unified dynamics or separate control? Acta Psychol (Amst) 117: 121-138, 2004.

Wigmore V, Tong C, Flanagan JR. Visuomotor rotations of varying size and direction compete for a single internal model in motor working memory. $J$ Exp Psychol 28: 447-457, 2002.

Wolpert DM, Kawato M. Multiple paired forward and inverse models for motor control. Neural Netw 11: 1317-1329, 1998. 